\title{
Linear ATC Enhancement with FACTS Devices Considering Reactive Power Flows using PTDF
}

\author{
Poornima Pankajam. T \\ Electrical Engineering Dept. \\ QIS college of Engg \& Tech. \\ Ongole, India
}

\author{
J. Srinivasa Rao \\ Electrical Engineering Dept \\ QIS college of Engg \& Tech. \\ Ongole, India
}

\author{
J. Amarnath \\ Electrical Engineering Dept. \\ JNTUH college of Engineering \\ Hyderabad, India
}

\begin{abstract}
In the present day world power system deregulation is at its full stretch. In this deregulated environment there is a clear need for adequate computation of ATC which is currently being given at most importance. The insertion of FACTS devices in electrical systems seems to be a promising strategy to enhance ATC. In this paper, the viability and technical merits of boosting ATC using TCSC are analyzed. The methods used for determining ATC are linear methods, which are based on MVA loading of the system considering system thermal limit constraints, neglecting bus voltages and static collapse. Power Transfer Distribution Factors, commonly referred to as PTDFs, express the percentage of a power transfer that flows on a transmission facility. They are used to determine the maximum ATC that may be available across the system without violating line thermal limits. The effect of reactive power flows in line loading is not considered in linear ATC which is a major limitation. This paper describes a fast algorithm to incorporate this effect. In this paper the line post transfer complex flow is estimated based on exact circle equation and then ATC is evaluated using active power distribution factors. The effectiveness of the proposed method is successfully demonstrated on IEEE 30-Bus system.
\end{abstract}

\section{Keywords}

Linear ATC, ACPTDF, DCPTDF, TCSC, Reactive Power.

\section{INTRODUCTION}

The electricity market, trade and operation, in the deregulated environment requires ample transmission capability to satisfy the demand of increasing power transactions. The conflict of this requirement and the restrictions on the transmission expansion in the restructured electricity market has motivated the development of methodologies to enhance the available transfer capability (ATC) of the existing transmission grids [4]. Transfer capability is the measure of the ability of interconnected electric systems to reliably move or transfer electric power from one area to another area, by way of all transmission lines or paths between those areas, under specified system conditions. The units of transfer capability are in terms of electric power, generally expressed in MWs. According to the North American Electric Reliability Council (NERC) definition, ATC is "a measure of the transfer capability remaining in the physical transmission network for further commercial activities over and above already committed uses".

The ATC value is given as: $\mathrm{ATC}=\mathrm{TTC}-\mathrm{TRM}-\{\mathrm{ETC}+\mathrm{CBM}\}$ Where TTC is total transfer capability, TRM is transmission reliability margin, CBM is capacity benefit margin and ETC is existing transmission commitment including customer services $[2,3]$.
The available transfer capability indicates the amount by which interarea bulk power transfers can be increased without compromising system security. The value used for available transfer capability affects both system security and the profits made in bulk power transactions. The transfer direction is a real power transaction which takes place between bus s (slack bus) and bus i while holding all other power flow injections fixed at their base case levels. Although the maximum secure transfer should be determined using full nonlinear computations (considering both static and dynamic constraints), ATC studies often involve contingencies and multipattern scenarios, making this an alarming task for most real systems [1]. The linear methods used for determining ATC introduce a number of potential errors. One of the limitations is ignoring reactive power flows [6]. This paper proposes an improved fast method to compute ATC incorporating reactive power flows. Also AC Power Transfer Distribution Factors (ACPTDFs) and DC Power Transfer Distribution Factors (DCPTDFs), for the fast determination of ATC using thermal limits and voltage limits, are proposed in this paper.

Flexible AC transmission systems (FACTS) controllers have been mainly used for solving various power system steady state control problems. They are power electronic based compensating devices and are known for their ability to enhance power transfer capability [8-11].

\section{ATC ASSESSMENT}

\subsection{Linear Method For Determining ATC:}

The ATC between two areas provides an indication of the amount of additional electric power that can be transferred from one area to another for a specific time frame for a specific set of conditions. ATC can be a very dynamic quantity because it is a function of variable and interdependent parameters. These parameters are highly dependent upon the conditions of the network. Consequently, ATC calculations may need to be periodically updated. Because of the influence of conditions throughout the network, the accuracy of the ATC calculation is highly dependent on the completeness and accuracy of available network data [4-6].Linear ATC typically assumes a lossless system, where changes in real power flow is linearly related to changes in power injections. For illustration, let us assume a transfer from the slack bus $l$, to any bus $m$, and maximize this transfer without exceeding any line or transformer. The key to the linear power solution is the use of Power Transfer Distribution Factors (PTDF) expressed here as sensitivities of line real power injections $[1,2]$. These PTDFs are essentially current dividers in linear circuit theory. As such, they are largechange sensitivities and can be used to predict the change in the line flow (line $p-n)$ due to a transfer bus $(l)$ to bus $(j)$ as 


$$
\begin{gathered}
\rho_{p n, m}=\frac{\partial P_{p n}}{\partial P_{l}}=-\frac{\partial P_{p n}}{\partial P_{m}} \\
\Delta P_{p n}=\rho_{p n, m}=-\rho_{p n, m} \Delta P_{m}
\end{gathered}
$$

Note that $\Delta P_{l}=\Delta P_{m}$ is the amount of transferred power from slack to any other bus. For a given positive line $P_{p n}^{\max }$, flow limit, assumed equal to the line MVA rating, and an initial positive line flow $P_{p n}^{0}$, the size of the transfer that drives the line to its limit is equal to

$$
\Delta P_{l}^{p n}=\left\{\begin{array}{cc}
\frac{P_{p n}^{\max }-P_{p n}^{0}}{\rho_{p n, m \rightarrow j}} & \rho_{p n, m}>0 \\
\frac{-P_{p n}^{\max }-P_{p n}^{0}}{\rho_{p n, m \rightarrow j}} & \rho_{p n, m}<0
\end{array}\right.
$$

In order to determine ATC, the minimum value of $\Delta P_{l}^{p n}$ among all lines in the system is determined [1].

$$
A T C_{l \rightarrow j}=\min \left\{\Delta P_{l}^{p n}: \text { all lines }(p-n)\right\}
$$

Note that it is the linear relation between the transfer and the line flows that makes linear ATC the fastest algorithm for transfer studies [1].

\subsection{Reactive Power Consideration In Linear ATC:}

The transmission line complex flow is constrained to be on the operating circle and inside the limiting circle, as such, the maximum complex flow of the line $p-n$ corresponds to point $\left(P_{p n}^{*}, Q_{p n}^{*}\right)$. Two different solutions for $P_{p n}^{*}$ can be obtained depending on the sign of the distribution factor. In order to compute $P_{p n}^{*}$ and $Q_{p n}^{*}$ the following system of equations must be solved;

$$
P_{p n}^{2}+Q_{p n}^{2}=\left(S_{p n}^{\max }\right)^{2}
$$

The following operating circle equation is obtained after incorporating various equations:-

$$
\left(P_{p n}-P_{i j \Theta}\right)^{2}+\left(Q_{p n}-Q_{i j \Theta}\right)^{2}=S_{p n \Theta}^{2}
$$

Expanding Eqn. (5) and then subtracting it from Eqn. (6), gives

$$
\begin{aligned}
Q_{p n}=\left(\frac{1}{2} Q_{p n \Theta}\right)( & -2 P_{i j} P_{p n \Theta}+\left(S_{p n}^{\max }\right)^{2} \\
& \left.-\left(S_{p n \Theta}^{2}-P_{p n \Theta}^{2}-Q_{p n \Theta}^{2}\right)\right)
\end{aligned}
$$

Let us assume $S_{p n \Theta}^{2}-P_{p n \Theta}^{2}-Q_{p n \Theta}^{2}$ as $\mathrm{T}$ and now the above Eqn. (7) becomes

$$
Q_{p n}=\left(\frac{1}{2} Q_{p n e}\right)\left(-2 P_{p n} P_{p n e}+\left(S_{p n}^{\max }\right)^{2}-\left(T^{2}\right)\right)
$$

The quadratic expression in $P_{p n}^{*}$ is obtained on substituting Eqn. (8) in Eqn. (5) and then replacing $P_{p n}$ by $P_{p n}^{*}$, as:-

$$
\begin{aligned}
P_{p n}^{* 2}\left(P_{p n \Theta}^{2}+Q_{p n \Theta}^{2}\right) & -P_{p n}^{*}\left[\left(\left(S_{p n}^{\max }\right)^{2}-T^{2}\right) P_{p n \Theta}\right] \\
& +\frac{1}{4}\left(\left(S_{p n}^{\max }\right)^{2}-T^{2}\right)^{2}-Q_{p n \Theta}^{2}\left(S_{p n}^{\max }\right)^{2} \\
& =0
\end{aligned}
$$

Defining the corresponding constant coefficients:-

$$
a=P_{p n b \Theta}^{2}+Q_{p n \Theta}^{2}
$$

$$
\begin{gathered}
b=-P_{p n}^{*}\left(\left(S_{p n}^{\max }\right)^{2}-T^{2}\right) \\
c=\frac{1}{4}\left(\left(S_{p n}^{\max }\right)^{2}-M^{2}\right)^{2}-Q_{p n \Theta}^{2}\left(S_{p n}^{\max }\right)^{2}
\end{gathered}
$$

Then the solution for the maximum complex flow is obtained as:-

$$
\begin{gathered}
P_{p n}^{*}=\frac{-b \pm \sqrt{b^{2}-4 a c}}{2 a} \\
Q_{p n}^{*}=\sqrt{\left(S_{p n}^{\max }\right)^{2}+P_{p n}^{* 2}}
\end{gathered}
$$

The sign in the previous equation is chosen to be positive if the PTDF of line $p-n$ is positive and negative otherwise [1].

$$
\Delta P_{l}^{p n}=\left\{\begin{array}{lc}
\frac{P_{p n}^{\max }-P_{p n}^{0}}{\rho_{p n, m \rightarrow j}} & \rho_{p n, m \rightarrow j}>0 \\
\frac{-P_{p n}^{\max }-P_{p n}^{0}}{\rho_{p n, m \rightarrow j}} & \rho_{p n, m \rightarrow j}<0
\end{array}\right.
$$

The process of computing linear ATC including the effect of reactive flows is summarized as follows:

Firstly compute the distribution factors $\rho_{p n, m}$ and then $P_{p n}^{*}$ using Eqn. (10) and Eqn. (11a). Secondly compute the necessary transfer $\Delta P_{l}^{p n}$ using Eqn. (12) or (13) then the minimum $\Delta P_{l}^{p n}$ among all line ends is obtained.

Since the incorporation of reactive flows into the algorithm resides in computing a new line flow limit, all of the advantages of the linear ATC method are preserved [1]. Therefore, the basic ATC problem regarding thermal security limits is given by an initial operating state of the power system, determine the maximum amount $\Delta P$ for a transaction between seller $(s)$ and buyer $(b)$ such that $\left|S_{p n}\right|<\left|S_{p n}^{\max }\right|$ for all $p-n$ lines in the system [5].

It should be remarked that the value of the ATC with the Reactive Linear method is always smaller than that value of the ATC with the Linear method, which can be considered as a conservative characteristic of the method [6].

\subsection{Computation Of PTDFs:}

PTDFs determine the linear impact of a transfer on the elements of the power system. These values provide a linearized approximation of how the flow on the transmission lines and interfaces change in response to transactions between the seller and buyer [2].

\subsubsection{DCPTDF Formulation:}

The linear DCPTDFs, based on DC power-flow equations, are used to allocate MW flows on the lines for a transaction in the system. These equations are simply the real part of decoupled power-flow equations. So only angle and real powers are solved by iterating

$$
[\Delta \delta]=[B]^{-1} \Delta P
$$

The net change in angle is

$$
\Delta \delta=\Delta \delta^{0}-\Delta \delta_{m n}
$$

Where $\Delta \delta^{0}$ is the base-case angle.

The power flow on lines $i$ and $j$ is given by

$$
P_{i j, m n}=\left(\frac{1}{x_{i j}}\right)\left(\Delta \delta_{i}-\Delta \delta_{j}\right) m
$$


Volume 76-No.4, August 2013

Where $x_{i j}$ denotes the reactance of the line $i-j$. However, this has a poor accuracy due to the assumption involved in the DC power flow model [8].

\subsubsection{ACPTDF Formulation:}

The ACPTDFs proposed for the calculation of ATC were used to find various transmission system quantities for a change in MW transaction at different operating conditions. Consider a bilateral transaction $t_{k}$ between a seller bus $m$ and buyer bus $n$. Line 1 carries the part of the transacted power and is connected between buses $i$ and $j$. PTDFs can be defined as

$$
P T D F_{i j, m n}=\frac{\Delta q_{1}}{\Delta t_{k}}
$$

The transmission quantity $\mathrm{q}_{1}$ can be either real power flow from bus $\mathrm{i}$ to $\mathrm{j}$ or $\mathrm{j}$ to $\mathrm{i}\left(\mathrm{P}_{\mathrm{ji}}\right)$. The above factors have been proposed to compute at a base-case load flow results using the sensitivity properties of NRLF Jacobian. Consider full Jacobian in polar coordinates $\left[J_{T}\right]$, defined to include all the buses except slack.

$$
\left[\begin{array}{l}
\Delta \delta \\
\Delta V
\end{array}\right]=\left[\begin{array}{ll}
\frac{\partial P}{\partial \delta} & \frac{\partial P}{\partial V} \\
\frac{\partial Q}{\partial \delta} & \frac{\partial Q}{\partial V}
\end{array}\right]^{-1}\left[\begin{array}{l}
\Delta P \\
\Delta Q
\end{array}\right]=\left[J_{T}\right]^{-1}\left[\begin{array}{c}
\Delta P \\
\Delta Q
\end{array}\right]
$$

In the above equation the change in active power flow of line $i-j$ with respect to changes in state variables is determined as

$$
\begin{aligned}
& \frac{\partial P_{i j}}{\partial \delta_{e}}=\left\{\begin{array}{cc}
0 & \text { for } e \neq i, j \\
-V_{i} V_{j} Y_{i j} \sin \left(\delta_{i}-\delta_{j}-\theta_{i j}\right) & \text { for } e=i \\
-V_{i} V_{j} Y_{i j} \sin \left(\delta_{i}-\delta_{j}-\theta_{i j}\right) & \text { for } e=j
\end{array}\right\} \\
& \frac{\partial P_{i j}}{\partial V_{e}} \\
& =\left\{\begin{array}{cc}
0 & \text { for } e \neq i, j \\
2 V_{i} Y_{i j}+V_{j} Y_{i j} \cos \left(\delta_{i}-\delta_{j}-\theta_{i j}\right) & \text { for } e=i \\
V_{j} Y_{i j} \cos \left(\delta_{i}-\delta_{j}-\theta_{i j}\right) & \text { for } e=j
\end{array}\right\}
\end{aligned}
$$

In a base-case load flow, if only one of the kth bilateral transactions is changed by $\Delta \mathrm{t}_{\mathrm{k}} \mathrm{MW}$, only the following two entries in the mismatch vector on right-hand side of Eqn. (18) will be non zero:

$\Delta P_{i}=\Delta t_{k}, \quad \Delta P_{j}=-\Delta t_{k}$

The new voltage profile can be calculated from Eqns. (18) and (21). These can be utilized to compute all of the transmission quantities $\mathrm{q}_{1}$ and hence corresponding quantities $\Delta \mathrm{q}_{1}$ from the base case. Once the $\Delta \mathrm{q}_{1}$ for all lines corresponding to a change in the transaction $\Delta t_{k}$ is known, the PTDFs can be obtained from Eqn. (17) [7].

\section{TCSC MODEL}

FACTS devices such as thyristor controlled series compensators controls the power flow in the network, and helps to reduce the flows in heavily loaded lines. The thyristor-controlled group employs capacitor and reactor banks with fast solid-state switches in traditional shunt or series circuit arrangements. The thyristor switches control the on and off periods of the fixed capacitor and reactor banks and thereby realize a variable reactive impedance. Figure 1 shows a model of a transmission line with a TCSC connected between buses $i$ and $j$. The transmission line is represented by its lumped $\Pi$ equivalent parameters connected between the two buses. During the steady state, the TCSC can be considered as a static reactance $-j X_{c}$. This controllable reactance, $X_{c}$, is directly used as the control variable to be implemented in the power flow equation $[2,9,10]$.

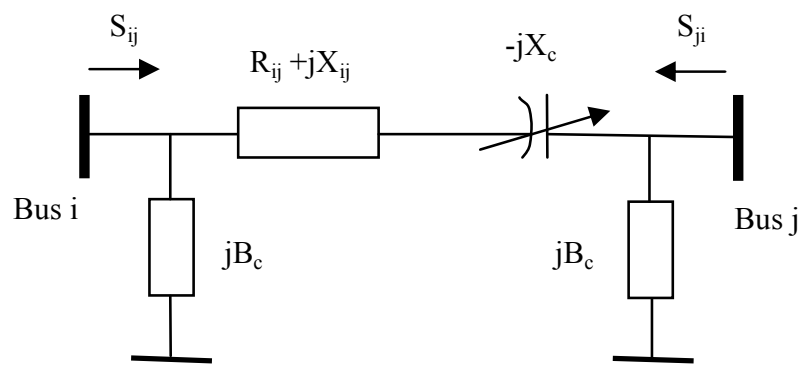

Fig. 1: Model Of TCSC

The following equations are used to model TCSC. The complex power from bus $i$ to $j$ is

$$
\begin{aligned}
& S_{i j}^{*}=P_{i j}-Q_{i j}=V_{i}^{*} I_{i j}=V_{i}^{*}\left[\left[G_{i j}+j\left(B_{i j}+B_{c}\right)\right]-\right. \\
& \left.V_{i}^{*} V_{j}\left(G_{i j}+j B_{i j}\right)\right]
\end{aligned}
$$

From the above equations the real and reactive power equations can be written as

$$
\begin{gathered}
P_{i j}= \\
V_{i}^{2} G_{i j}-V_{i} V_{j} G_{i j} \cos \left(\delta_{i}-\delta_{j}\right)-V_{i} V_{j} B_{i j} \sin \left(\delta_{i}-\delta_{j}\right) \\
Q_{i j}=-V_{i}^{2}\left(B_{i j}+B_{c}\right)-V_{i} V_{j} G_{i j} \sin \left(\delta_{i}-\delta_{j}\right)+ \\
V_{i} V_{j} B_{i j} \cos \left(\delta_{i}-\delta_{j}\right)
\end{gathered}
$$

\section{SENSITIVITY APPROACH FOR OPTIMAL PLACEMENT OF TCSC}

Generally, the location of FACTS devices depends on the objective of the installation. In this paper the main objective considered is to enhance ATC. Consider a line connected between buses $m$ and $n$ and having a net series impedance of $X m n$, that includes the reactance of a TCSC, if present, in that line[9]. The loss sensitivities with respect to Xmn can be computed as:

$a_{m n}=\frac{\partial Q_{L}}{\partial X_{m n}}=$
$\left[V_{m}^{2}+V_{n}^{2}-2 V_{m} V_{n} \cos \left(\delta_{m}-\delta_{n}\right)\right] \frac{R_{m n}^{2}-X_{m n}^{2}}{\left(R_{m n}^{2}+X_{m n}^{2}\right)}$

Where, $V_{m}$ is the voltage at bus $\mathrm{m}$,

$V_{n}$ is the voltage at bus $\mathrm{n}$,

$R_{m n}$ is resistance of line between bus $\mathrm{m}$ and $\mathrm{n}$,

$X_{m n}$ is the reactance connected between bus $\mathrm{m}$ and $\mathrm{n}$.

With the sensitive indices computed for FACTS device, TCSC, should be placed in the most positive line [11].

\section{SIMULATION AND RESULTS}

A sample 30-bus system is considered to illustrate the implementation of TCSC for ATC enhancement. The simulation has been carried out for the IEEE 30 bus system, by incorporating FACTS device (i.e. TCSC), using Power World Simulator Software. 


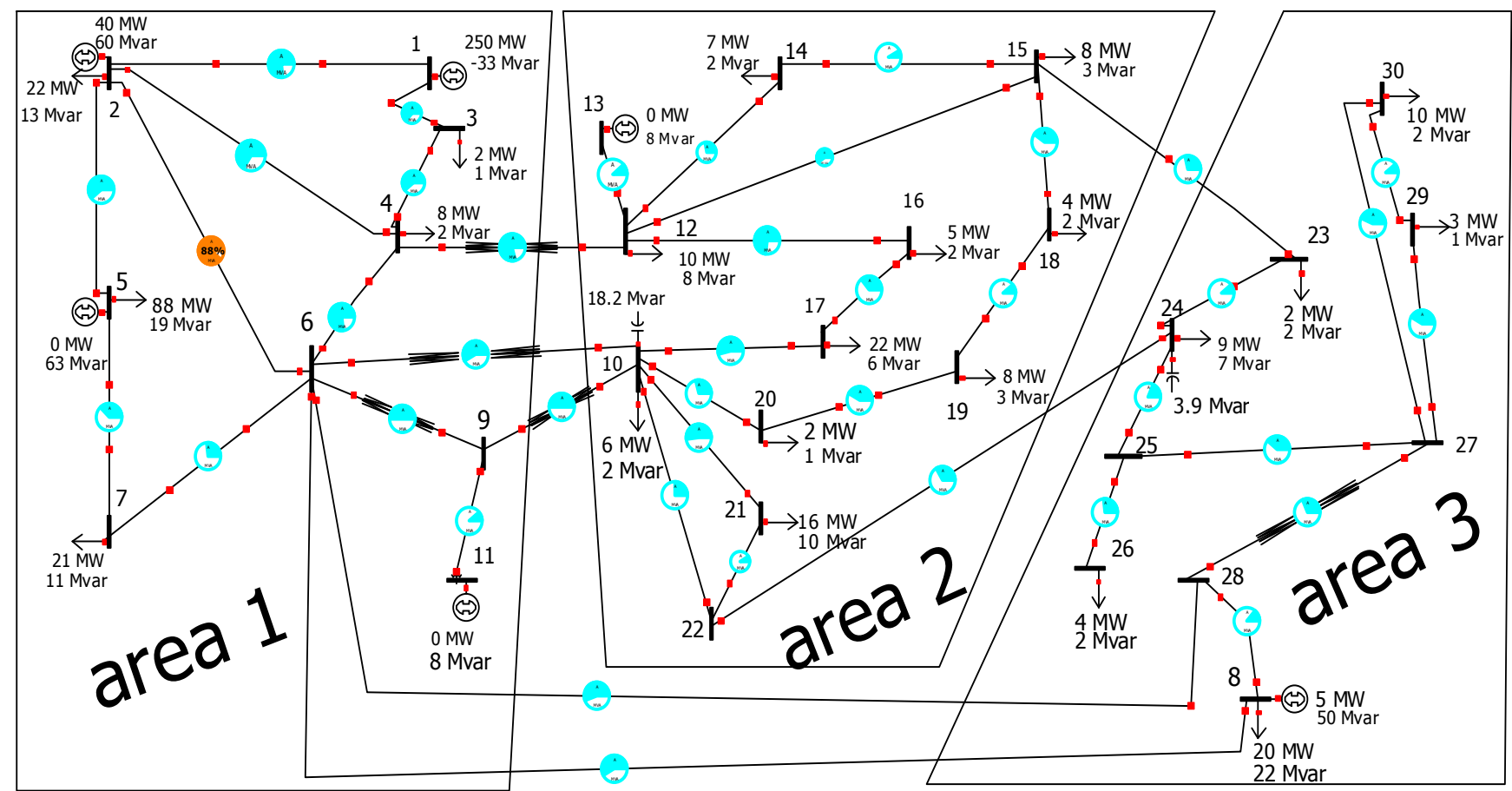

Fig 2: Modified IEEE 30 bus system

The single line diagram of the modified IEEE-30 bus system is shown in figure 2 . The system is divided in to three areas. The ATC is calculated between all the areas. Base values are assumed to be 100 MVA. The ATC enhancement results for the 30 bus system, ignoring and considering reactive power flows can be observed from the below graph:

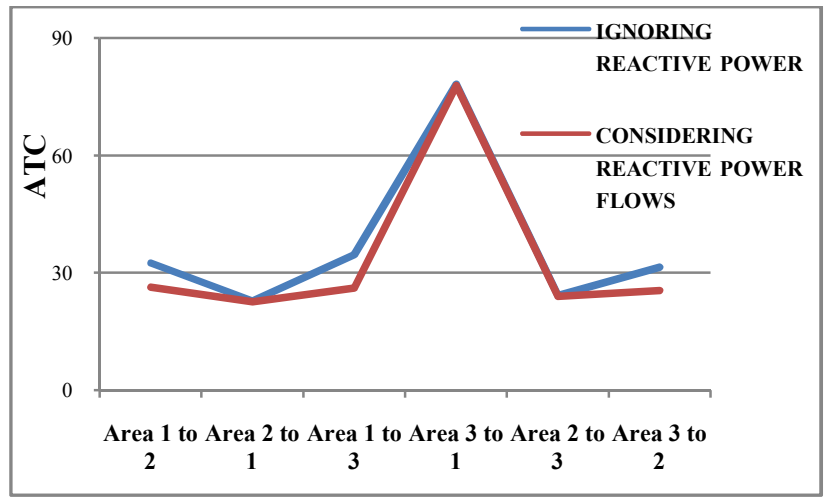

Fig 3 : Available Transfer Capability (ATC)

The proposed PTDFs can be computed quite quickly at base case load flow results and can be utilized for changes in system operating conditions. Use of these factors offer an approximate but extremely fast model for the static ATC determination. However DCPTDFs are derived based on DC load flow assumptions and hence provide less accurate results when compared to ACPTDFs. The ATC using ACPTDF and DCPTDF methods for ignoring and considering reactive power flows is plotted below.

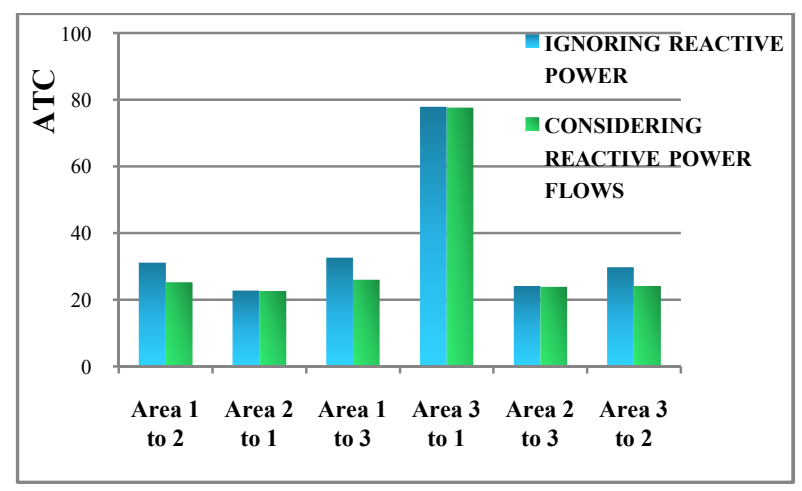

Fig 4: ATC using ACPTDF

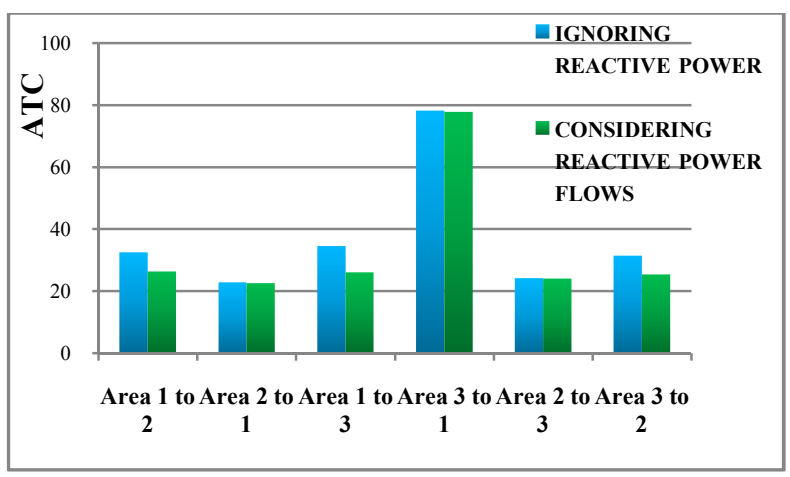

Fig 5: ATC using DCPTDF 
The FACTS device considered here is Thyristor Controlled Series Capacitor (TCSC). By using sensitivity approach analysis, the optimal placement of TCSC is determined. The most positive sensitivity in case of TCSC is highlighted in the tabular form. The TCSC is located in the line connected between buses 14 and 15. The sensitivity indices for TCSC at different compensation levels is tabulated in the table 1 .

Table 1: Sensitivity Factors For TCSC

\begin{tabular}{|c|c|c|c|c|c|}
\hline \multirow[t]{2}{*}{ Line } & \multirow{2}{*}{$\begin{array}{c}\text { From } \\
\text { Bus }\end{array}$} & \multirow{2}{*}{$\begin{array}{c}\text { To } \\
\text { Bus }\end{array}$} & \multicolumn{3}{|c|}{ Sensitivity Index } \\
\hline & & & $\begin{array}{c}\text { TCSC(2 } \\
\mathbf{0} \%) \\
\left(\mathbf{a}_{\mathrm{ij}}\right)\end{array}$ & $\begin{array}{c}\operatorname{TCSC}(4 \\
0 \%)\left(a_{i j}\right)\end{array}$ & $\begin{array}{l}\operatorname{TCSC}(6 \\
0 \%)\left(a_{i j}\right)\end{array}$ \\
\hline 1 & 1 & 2 & -2.9812 & -3.5610 & -2.0961 \\
\hline 2 & 1 & 3 & -0.8416 & -1.1476 & -1.1747 \\
\hline 3 & 2 & 4 & -0.1973 & -0.2390 & -0.1513 \\
\hline 4 & 2 & 5 & -0.8124 & -1.1811 & -1.4882 \\
\hline 5 & 2 & 6 & -0.3506 & -0.4234 & -0.2634 \\
\hline 6 & 3 & 4 & -0.6649 & -0.7656 & -0.3633 \\
\hline 7 & 4 & 6 & -0.6010 & -0.7972 & -0.7401 \\
\hline 8 & 4 & 12 & -0.3431 & -0.61004 & -1.3726 \\
\hline 9 & 5 & 7 & -0.0653 & -0.0652 & -0.0023 \\
\hline 10 & 6 & 7 & -0.1458 & -0.1777 & -0.1159 \\
\hline 11 & 6 & 8 & -0.1062 & -0.1414 & -0.1330 \\
\hline 12 & 6 & 9 & -0.1777 & -0.3160 & -0.7110 \\
\hline 13 & 6 & 10 & -0.0536 & -0.0953 & -0.2144 \\
\hline 14 & 6 & 28 & -0.0393 & -0.0527 & -0.0508 \\
\hline 15 & 8 & 28 & -0.0033 & -0.0042 & -0.0029 \\
\hline 16 & 9 & 10 & -0.1708 & -0.3037 & -0.6832 \\
\hline 17 & 9 & 11 & -0.0091 & -0.0162 & -0.0364 \\
\hline 18 & 10 & 17 & -0.0287 & -0.0299 & -0.0049 \\
\hline 19 & 10 & 20 & -0.0063 & -0.0051 & 0.0032 \\
\hline 20 & 10 & 21 & -0.0196 & -0.0147 & 0.0134 \\
\hline 21 & 10 & 22 & -0.0041 & -0.0027 & 0.0037 \\
\hline 22 & 12 & 13 & -0.0091 & -0.0161 & -0.0362 \\
\hline 23 & 12 & 14 & -0.0051 & -0.0035 & -0.0044 \\
\hline 24 & 12 & 15 & -0.0214 & -0.0121 & 0.0253 \\
\hline 25 & 12 & 16 & -0.0094 & -0.0066 & 0.0076 \\
\hline 26 & 14 & 15 & 0.000059 & 0.00012 & 0.00019 \\
\hline 27 & 15 & 18 & -0.0028 & -0.0018 & 0.0028 \\
\hline 28 & 15 & 23 & -0.0014 & -0.0009 & 0.0015 \\
\hline 29 & 16 & 17 & -0.0040 & -0.0055 & -0.0057 \\
\hline 30 & 18 & 19 & -0.00024 & -0.00015 & 0.00024 \\
\hline 31 & 19 & 20 & -0.0028 & -0.0017 & 0.0031 \\
\hline 32 & 21 & 22 & -0.0004 & -0.00023 & 0.00036 \\
\hline 33 & 22 & 24 & -0.00087 & 0.00037 & 0.0033 \\
\hline 34 & 23 & 24 & -0.00025 & -0.00016 & 0.00024 \\
\hline 35 & 24 & 25 & -0.00039 & -0.00008 & 0.00083 \\
\hline 36 & 25 & 26 & -0.00047 & 0.00039 & 0.0022 \\
\hline 37 & 25 & 27 & -0.0025 & -0.0012 & 0.0034 \\
\hline 38 & 28 & 27 & -0.0613 & -0.1090 & -0.2453 \\
\hline 39 & 27 & 29 & -0.0026 & -0.0012 & 0.0038 \\
\hline 40 & 27 & 30 & -0.0028 & -0.0012 & 0.0042 \\
\hline 41 & 29 & 30 & -0.00057 & -0.00026 & 0.00083 \\
\hline
\end{tabular}

The ATC results of the system without and with TCSC, at different compensation levels, for a modified IEEE-30 bus system can be observed from the tables below. Table 2 represents ATC results for ignoring reactive power and Table 3 for considering reactive power. The value of the ATC is enhanced considerably by placing TCSC in the system.
Table 2: ATC Ignoring Reactive Power

\begin{tabular}{|c|c|c|c|c|}
\hline $\begin{array}{c}\text { From } \\
\text { Area To } \\
\text { Area }\end{array}$ & $\begin{array}{c}\text { ATC } \\
\text { Without } \\
\text { FACTS }\end{array}$ & $\begin{array}{c}\text { ATC } \\
\text { With } \\
\text { TCSC(20 } \\
\text { \%) }\end{array}$ & $\begin{array}{c}\text { ATC } \\
\text { With } \\
\text { TCSC(4 } \\
\text { 0\%) }\end{array}$ & $\begin{array}{c}\text { ATC } \\
\text { With } \\
\text { TCSC(6 } \\
\text { 0\%) }\end{array}$ \\
\hline $1-2$ & 32.56 & 32.57 & 32.58 & 32.6 \\
\hline $2-1$ & 22.86 & 22.94 & 23.03 & 23.12 \\
\hline $1-3$ & 34.63 & 34.64 & 34.64 & 34.64 \\
\hline $3-1$ & 78.23 & 78.23 & 78.23 & 78.23 \\
\hline $2-3$ & 24.27 & 24.36 & 24.45 & 24.56 \\
\hline $3-2$ & 31.48 & 31.49 & 31.5 & 31.51 \\
\hline
\end{tabular}

Table 3: ATC Considering Reactive Power Flows

\begin{tabular}{|c|c|c|c|c|}
\hline $\begin{array}{c}\text { From } \\
\text { Area To } \\
\text { Area }\end{array}$ & $\begin{array}{c}\text { ATC } \\
\text { Without } \\
\text { FACTS }\end{array}$ & $\begin{array}{c}\text { ATC } \\
\text { With } \\
\text { TCSC(20 } \\
\text { \%) }\end{array}$ & $\begin{array}{c}\text { ATC } \\
\text { With } \\
\text { TCSC( } \\
\mathbf{4 0 \% )}\end{array}$ & $\begin{array}{c}\text { ATC } \\
\text { With } \\
\text { TCSC(6 } \\
\text { 0\%) }\end{array}$ \\
\hline $1-2$ & 26.36 & 26.37 & 26.37 & 26.38 \\
\hline $2-1$ & 22.65 & 22.72 & 22.81 & 22.91 \\
\hline $1-3$ & 26.12 & 26.12 & 26.12 & 26.12 \\
\hline $3-1$ & 77.87 & 77.87 & 77.87 & 77.87 \\
\hline $2-3$ & 24.05 & 24.13 & 24.22 & 24.33 \\
\hline $3-2$ & 25.49 & 25.49 & 25.5 & 25.51 \\
\hline
\end{tabular}

The plots which represent the lodabilty of transmission lines at different compensation levels were shown in figures 6 and 7 . From the plots below it can be clearly observed that by placing TCSC in the most positive lines, the power carrying capability of most of the transmission lines increases.

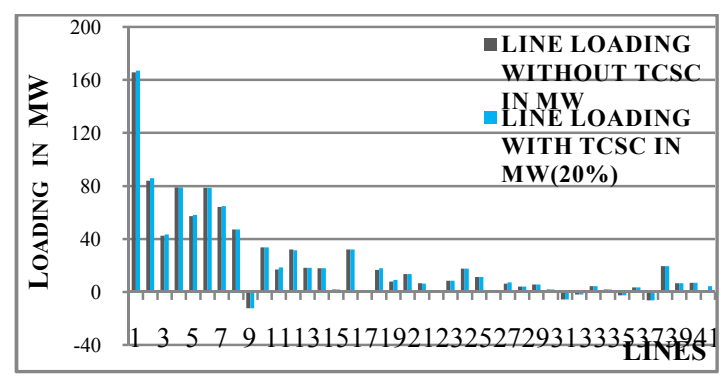

Fig 6: Loadability Of Lines At 20\% Compensation

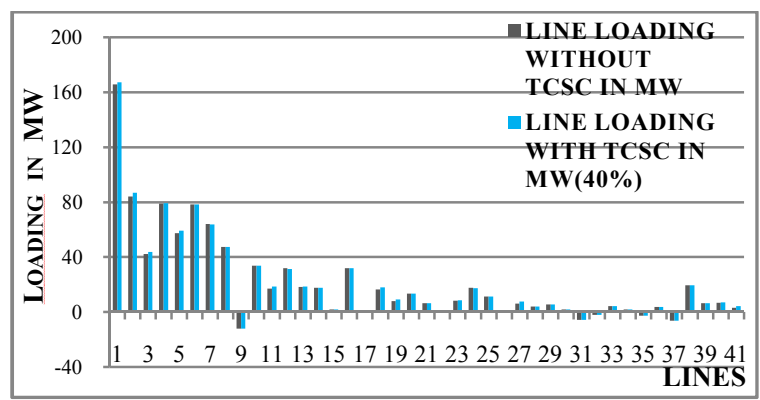

Fig 7: Loadability Of Lines At 40\% Compensation 


\section{CONCLUSION}

An accurate ATC computation is also very important to the transmission system. If the computed ATC is less than the ATC of the system, the transmission of power will not be efficient economically, if the computed ATC is more than the ATC of the system, the transmission will be operating in a dangerous state and any power increased will stand a chance to collapse the whole system and the result of that is disastrous. The results obtained in this paper by incorporating the effect of reactive power flows in transmission elements show a significant error reduction in linear ATC. The method is based on a megavarcorrected megawatt limit, which captures the change in reactive power flow as the active power flow in the line increases due to large transfers. This method can be easily incorporated into existing linear ATC software. Also ATC is calculated using AC and DC power transfer distribution factors. The inclusion of reactive power and FACTs device in a linear ATC can enhance the maximum transaction over a transmission system.

\section{REFERENCES}

[1] S. Grijalva W. Sauer, J. D. Weber, "Enhancement of Linear ATC Calculations by the Incorporation of Reactive Power Flows", IEEE Transactions on Power Systems, Vol. 18, No. 2, pp.619-624, May 2003.

[2] Ibraheem and Naresh kumar Yadav, "Implementation OF FACTS Device For Enhancement Of ATC Using PTDF", International Journal of Computer and Electrical Engineering, Vol.3, No.3, June 2011.

[3] "Available Transfer Capability Definitions and Determination", North American Electric Reliability Council, Reference Document, June 1996.

[4] B.V. Manikandan, S. Charles Raja, P. Venkatesh, "Enhancement Of Available Transfer Capability With Facts Device In Competitive Power Market", Dec. 20-22, 2007. pp.50-56, IET-UK ICTES 2007.

[5] S.Grijalva, Peter W. Sauer, "Reactive Power Considerations in Linear ATC Computation", Decision Support Systems, Volume 30, Issue 3, pp. 327-340, January 2001.

[6] F. C. Gaino, and A. Padilha-Feltrin, "Influence of the Supply of Reactive Power in the Calculation of the Transfer Capability", 2004 IEEE/PES T \& D Conference 8 Exposition: Latin America.

[7] Dr. Paramasivam Venkatesh, Ramachandran Gnanadass, Dr. Narayana Prasad Padhy, "Available Transfer
Capability Determination Using Power Transfer Distribution Factors", International Journal Of Emerging Electric Power Systems, Vol 1, 2004.

[8] K. Radha Rani, J. Amarnath and S. Kamakshaiah , "Allocation Of FACTS Devices For ATC Enhancement Using Genetic Algorithm", ARPN Journal of Engineering and Applied Sciences, Vol. 6, No. 2, February 2011

[9] Y. Xiao, Y.H. Song, C.C. Liu and Y.Z. Sun, "Available Transfer Capability Enhancement using FACTS Devices", IEEE Transactions on Power systems, Vol.18, No.12, pp.305-312, February 2003.

[10] Wang Feng, G. B. Shrestha," Allocation of TCSC Devices to Optimize Total Transmission Capacity in a Competitive Power Market", IEEE Transactions on Power Systems, 2001.

[11] Narasimharao , J.Amarnath and K.Arun Kumar ,"Voltage Constrained Available Transfer Capability Enhancement With FACTS Devices", ARPN Journal of Engineering and Applied Sciences,vol 2, December 2007.

\section{AUTHORS}

POORNIMA PANKAJAM. T is pursuing M-Tech at QIS college of Engineering \& Technology under JNTU, Kakinada. She received her B-Tech degree from QISCET, Ongole under JNTU Kakinada. Her current research interests are FACTS applications on transmission systems, Power System Control and Automation.

SRINIVASARAO.J is an associate professor in QIS College of Engineering \& Technology at Ongole. He is pursuing Ph.D. He got his M-Tech degree from JNTU Hyderabad and his B-Tech degree from RVRJC Engineering College, Guntur. His current research interests are power systems, power systems control and automation, Electrical Machines, power systems deregulation, FACTS applications.

AMARNATH.J obtained the B.E degree in electrical engineering from O.U, Hyderabad and the M.E. degree in power systems from A.U, Visakhapatnam. Presently he is professor and head of the department of Electrical and Electronics engineering department, JNTU, Hyderabad. His research interests includes high voltage engineering, gas insulated substations, industrial drives, power electronics, power systems. 